\title{
Nonlinear Timing Jitter in an Installed Fiber Network With Balanced Dispersion Compensation
}

\author{
A. S. Lenihan, O. V. Sinkin, B. S. Marks, Member, IEEE, G. E. Tudury, R. J. Runser, Member, IEEE, A. Goldman, \\ C. R. Menyuk, Fellow, IEEE, and G. M. Carter, Senior Member, IEEE
}

\begin{abstract}
We present field measurements of timing jitter induced by intrachannel cross-phase modulation on an installed terrestrial fiber link. The measured jitter is strongly dependent on the arrangement of dispersion-compensating fiber, in excellent qualitative agreement with numerical simulations. We find that balancing the dispersion compensation yields significant benefits, even for an inhomogeneous fiber path. We show that numerical simulation combined with experimental results is a practical way to develop a link model, even when the system loss cannot be completely characterized experimentally.
\end{abstract}

Index Terms-Communication system nonlinearities, optical fiber communication, timing jitter, optical fiber dispersion.

\section{INTRODUCTION}

$\mathbf{I}_{\mathrm{i} \text { (1) }}$ NTRACHANNEL nonlinear effects can be a limiting factor in the performance of either single-channel or widely spaced wavelength-division-multiplexed fiber communications systems. In particular, the timing jitter caused by cross-phase modulation between adjacent bits can be a significant impairment for high-speed return-to-zero transmission. The effect of intrachannel timing jitter has been studied extensively in the context of dispersion-managed undersea transmission [1]-[7]. However, terrestrial systems, which may possibly include uncompensated spans or several different fiber types, have received less attention. Laboratory experiments performed on a recirculating loop indicate that for long-haul terrestrial transmission the management of the accumulated dispersion function is still necessary to minimize the timing jitter [8]. However, the consequences of concatenating several networks, each with its own amplifier distribution and dispersion variation, have not been addressed, but are of relevance in considering reconfigurable all-optical networks.

In this letter, we report on field measurements of intrachannel nonlinearity-induced timing jitter performed on an installed

Manuscript received December 22, 2004; revised March 7, 2005. This work was supported by the Laboratory for Telecommunication Sciences, by the Laboratory for Physical Sciences, by the National Science Foundation, by the Defense Advanced Research Projects Agency, and by the U.S. Department of Energy.

A. S. Lenihan, B. S. Marks, G. E. Tudury, and G. M. Carter are with the Department of Computer Science and Electrical Engineering, University of Maryland, Baltimore County, Baltimore, MD 21250 USA, and also with the Laboratory for Physical Sciences, College Park, MD 20740 USA (e-mail: lenihan@lps.umd.edu).

O. V. Sinkin and C. R. Menyuk are with the Department of Computer Science and Electrical Engineering, University of Maryland, Baltimore County, Baltimore, MD 21250 USA.

R. J. Runser is with Telcordia Technologies, Red Bank, NJ 07701 USA.

A. Goldman is with the Laboratory for Telecommunications Sciences, Adelphi, MD 20783 USA.

Digital Object Identifier 10.1109/LPT.2005.850009 fiber path. The fiber path consisted of segments from two different installed networks, which together serve to emulate two metropolitan access rings connected by a longer intercity link. Each of the two networks has its own characteristics for fiber type, dispersion compensation, and amplification, resulting in an inhomogeneous transmission path which is a useful prototypical system for studying these nonlinear effects in a realistic all-optical terrestrial network system. Our results indicate that management of the dispersion compensation will be important for achieving optimal performance in a reconfigurable high-speed optical network.

We also report on a link model that we created for the entire system. A practical difficulty that appears in field measurements, in contrast to laboratory measurements, is that important system parameters are often not fully known. In our case, the missing parameters included the noise figures of the span amplifiers and the location of excess system loss, beyond that associated with the fiber attenuation, which for some spans approached $10 \mathrm{~dB}$. While these unknown parameter values strongly impact the system performance, we have found that it is possible with fairly simple assumptions and limited numerical experimentation to obtain excellent agreement the experiments. Thus, the combination of the experiments and the simulations yields a practical approach for developing link models that can be used to design systems.

\section{EXPERIMENTAL SETUP}

We performed the measurements on the installed fiber path depicted in Fig. 1, which consisted of segments of two independent networks: the ATDnet ring in the Washington, DC area [9], and BOSSnet, which connects Washington, DC and Boston, MA [10]. The ATDnet fiber segment covered $\sim 43 \mathrm{~km}$ and was comprised of three fiber types: SMF-28 $(L=2.2 \mathrm{~km}, D=+17 \mathrm{ps} / \mathrm{nm} \cdot \mathrm{km}$ at $1550 \mathrm{~nm})$, TrueWave-RS $(L=40.6 \mathrm{~km}, D=+4.5 \mathrm{ps} / \mathrm{nm} \cdot \mathrm{km})$, and AllWave ( $L=0.3 \mathrm{~km}, D=+17 \mathrm{ps} / \mathrm{nm} \cdot \mathrm{km})$. After passing through ATDnet, the signal was amplified by a single erbium-doped fiber amplifier (EDFA) and then optically switched to a $\sim 16-\mathrm{km}$ segment of Allwave fiber, which linked the two networks. Neither of these paths were dispersion compensated within the link. The BOSSnet path consisted entirely of TrueWave Classic $(D=+2.8 \mathrm{ps} / \mathrm{nm} \cdot \mathrm{km})$ fiber. Amplifier huts were located at uneven intervals along the path, as indicated in Fig. 1. Each hut consisted of a pair of EDFAs, a gain-flattening filter, and dispersion-compensating fiber (DCF) to compensate for the dispersion of the preceding segment at $1550 \mathrm{~nm}$. The amplifiers operated in the saturated regime, with total output powers for all channels ranging from 0 to $6 \mathrm{dBm}$ into the following span. 


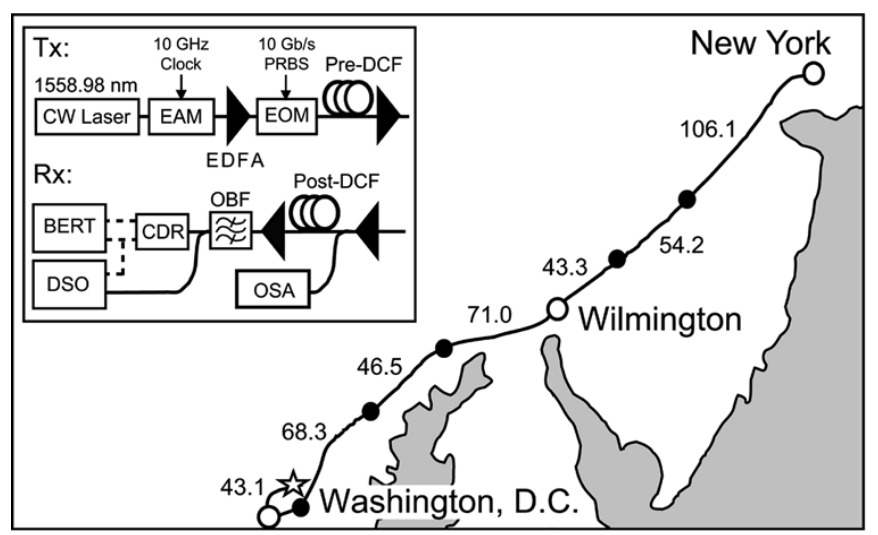

Fig. 1. Schematic of the installed fiber path. Circles indicate amplifier locations; open circles include loop-back capability. All distances are in kilometers. Inset: Diagram of the transmitter and receiver.

We utilized a single $10-\mathrm{Gb} / \mathrm{s}$ return-to-zero channel at $1558.983 \mathrm{~nm}$, using the transmitter and receiver shown in Fig. 1(inset). These were co-located at one of the ATDnet nodes in Adelphi, MD, indicated by the star in Fig. 1. We generated the test channel by pulse-carving a laser diode source, producing a $10-\mathrm{GHz}$ train of $\sim 30$-ps pulses which was then amplitude modulated with a $10-\mathrm{Gb} / \mathrm{s} 2^{31}-1$ pseudorandom bit sequence (PRBS). We launched the test channel into the network with an average power of $\sim 0 \mathrm{dBm}$. In order to focus on intrachannel effects, the test channel was separated by at least $600 \mathrm{GHz}$ from the other two signals on the networks: On ATDnet, a 1552.42-nm channel carried an ATM over SONET signal, while on BOSSnet a gigabit ethernet channel propagated at $1546.9 \mathrm{~nm}$.

At the receiver, we used a commercial $10-\mathrm{Gb} / \mathrm{s}$ receiver (CDR) to detect the amplified and optically filtered output signal. We monitored the recovered data and clock using a bit-error-rate (BER) tester. We used a digital sampling oscilloscope (DSO) with a 40-GHz bandwidth optical detection module to record the received eye diagrams, from which we extracted the signal timing jitter. In order to isolate the timing jitter that results from transmission, the inherent jitter of the oscilloscope, found in back-to-back measurements to be $\sim 1.1 \mathrm{ps,}$ was deconvolved from the results [8]. We also used the DSO eye diagram to calculate the signal $Q$-factor, from the means and standard deviations of the marks and spaces measured at an ideal sampling point. While the timing jitter will be more sensitive to the intrachannel cross-phase modulation, we utilized the $Q$-factor as a monitor to ensure that we were not incurring penalties from other nonlinearities, such as four-wave mixing, which can distort the eye.

We performed measurements for two transmission distances, by looping back the signal at two different locations along the BOSSnet path: Wilmington, DE and New York, NY. The return signal followed a parallel path, so that the total round-trip distances were 523 and $931 \mathrm{~km}$, respectively. Due to the lack of compensation on ATDnet and the detuning of the signal channel from the zero-dispersion wavelength of BOSSnet, significant dispersion was accumulated during transmission. In Fig. 2, we show the accumulated dispersion for each loop-back location, calculated with the specified fiber parameters. To compensate for this, we used additional DCF modules at the transmitter and
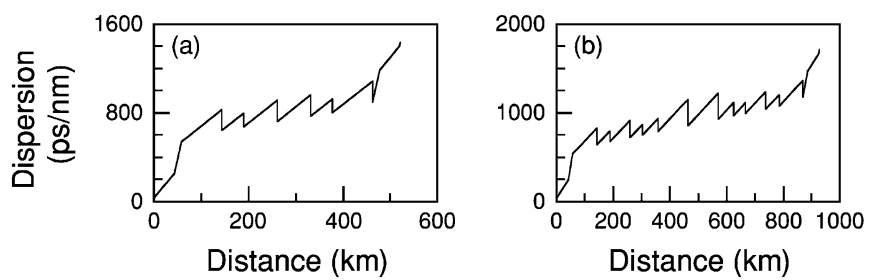

Fig. 2. Accumulated dispersion at $1558.98 \mathrm{~nm}$ as a function of propagation distance for the (a) Wilmington and (b) New York loop-backs.

the receiver. For each transmission distance, we experimentally chose the total amount of DCF by optimizing the system performance, as quantified by the scope $Q$-factor and timing jitter. We found the totals to be -1510 and $-1850 \mathrm{ps} / \mathrm{nm}$ for Wilmington and New York, respectively. We then kept this total quantity of DCF fixed, but varied the balance between precompensation and postcompensation while recording the received BER, optical signal-to-noise ratio, $Q$-factor, and timing jitter. The power into the precompensating and postcompensating DCF modules was kept low enough to prevent any additional nonlinearities from accumulating at the transmitter and receiver.

\section{NUMERICAL SIMULATIONS}

We compared the experimental timing jitter results with numerical simulations based on the nonlinear Schrödinger equation, including dispersion, loss, gain, and noise due to amplified spontaneous emission (ASE). We constructed the model of the transmission link using the known values of the fiber lengths, dispersion parameters, and loss coefficients. A nonlinear coefficient of $1.6 \times 10^{-20} \mathrm{~m}^{2} / \mathrm{W}$ was used for all of the fiber types except for DCF, for which we used $2.55 \times 10^{-20} \mathrm{~m}^{2} / \mathrm{W}$. Polarization effects such as polarization-mode dispersion were not included, as they were not found to impact the measurements. In addition, without access to each amplifier hut along the path, we were unable to measure the noise figure of each EDFA. Therefore, we matched the measured optical spectrum analyzer spectra at the receiver by adjusting the noise figure within a reasonable range of the specified values [10], along with typical values for the optical filter bandwidths. For each amount of precompensation, we propagated a signal with a PRBS of length 512 and used this to calculate the timing jitter. We found that for the present system, a maximum of only four pulses overlap; however, the longer pattern length was used to improve noise statistics.

For the loss of each span, we compared the calculated fiber loss with the powers measured at the input and output of each amplifier hut to determine the excess loss in the link. In some cases, the additional losses total nearly $10 \mathrm{~dB}$. The exact location of this additional loss can strongly impact the nonlinear-induced timing jitter, but experimentally it can be prohibitively difficult to accurately determine its position in such an installed system. However, the simulated timing jitter is sensitive to the placement of this loss both in its magnitude and in its dependence on the precompensation and postcompensation arrangement. We therefore divided this excess loss and located portions before and after the DCF at each amplifier node. The division of the loss between the two points was varied for each span, in order to obtain the best agreement with the experimental results for the timing jitter. The resulting input powers to the DCF 


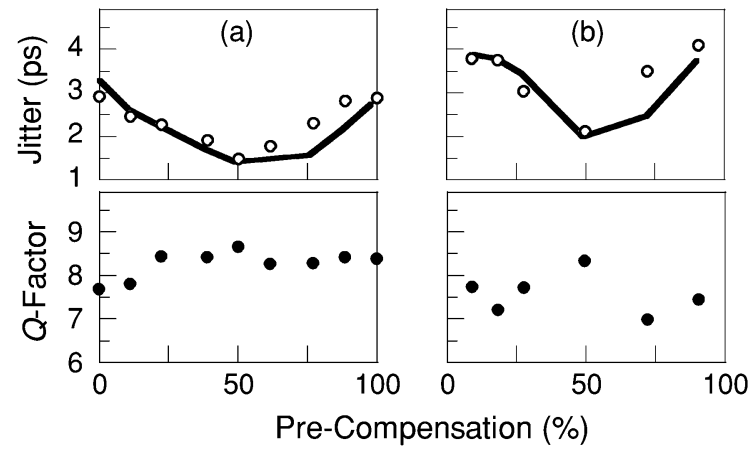

Fig. 3. Timing jitter and $Q$-factor (linear) for the (a) Wilmington and (b) New York loop-backs. Both the experimental (open circles) and theoretical (solid line) timing jitter values are shown.

ranged from -10 to $-3 \mathrm{dBm}$, depending on the particular link. This procedure is important because it can be used to create a link model, even in those cases when it is not practical to measure the exact location of the losses, such as in a large installed network.

\section{RESULTS}

In Fig. 3, we show the measured and simulated timing jitter, and the measured $Q$-factors, versus the ratio of precompensation to total dispersion compensation for (a) the Wilmington and (b) the New York loop-backs. At each distance, we observe significant variation in the timing jitter, while the $Q$-factors remain fairly constant. In all cases, the received signal was error-free $\left(\mathrm{BER} \leq 1 \times 10^{-9}\right)$. The $Q$-factors are significantly lower than the average back-to-back value of $\sim 18$, and are dominated by ASE from the link EDFAs, resulting in their insensitivity to the timing jitter. Further, as noted above we have taken care to avoid other nonlinear effects, which could distort the pulseshape and result in a variation in $Q$-factor with precompensation. From these results, we can conclude that the timing jitter variation is due to nonlinear effects, rather than amplitude jitter, which is known to contribute to timing jitter measurements [11]. For both loop-backs, the timing jitter is minimized near $50 \%$ precompensation. The numerical simulations agree quite well with the measured results and show the same qualitative features. We note that balancing the total DCF between the transmitter and receiver, as compared to placing it all at one end, proved effective in improving performance by minimizing jitter, even though all system parameters were not exactly known. The excellent agreement between experiment and simulation, obtained even without full knowledge of all the system parameters, demonstrates that the combination of the two provide a practical way to develop a link model.

With regards to the arrangement of dispersion compensation, it has been shown in [8] that the optimal precompensation should result in an accumulated dispersion function which is symmetric with respect to a $180^{\circ}$ rotation about the transmission midpoint. Here, we have demonstrated that this analysis extends to installed systems; as observed in Fig. 3, due in part to the symmetry imparted largely by the loopback, the optimal precompensation value is $\sim 50 \%$ of the total; however, we stress that for some systems the optimal balance may differ significantly from that observed here. Although the maximum timing jitter measured for nonoptimal precompensation, $\sim 4 \mathrm{ps}$ for the New York loop-back, is still within the tolerance range for $10 \mathrm{~Gb} / \mathrm{s}$, it reduces the performance margin available for other impairments, which may be environmental and random in nature. Therefore, it is important to maintain the optimal DCF balance in order to have the widest system margin, requiring one to characterize the possible paths within the network.

\section{CONCLUSION}

We have presented experimental and numerical results that demonstrate the need to properly arrange the distribution of dispersion compensation between the transmitter and the receiver, in order to achieve optimal performance in an installed optical network. In an all-optical network, where signals may be rerouted over different fiber spans, the characterization of the possible fiber pathways will be important in order to determine the appropriate compensation arrangement. For an already installed system, such characterization must often be done without full knowledge of important system parameters. We have shown that in such a situation, numerical simulation combined with experimental results is a practical way to develop an effective link model. Such models can then be used to estimate performance in a large optical network where considerable variations may exist between the different paths.

\section{ACKNOWLEDGMENT}

The authors thank T. Gibbons, T. Lehman, L. Mercer, and P. Schulz for their assistance in accessing the fiber links.

\section{REFERENCES}

[1] M. I. Hayee and A. E. Willner, "Pre- and post-compensation of dispersion and nonlinearities in $10 \mathrm{~Gb} / \mathrm{s}$ WDM systems," IEEE Photon. Technol. Lett., vol. 9, no. 9, pp. 1271-1273, Sep. 1997.

[2] P. V. Mamyshev and N. A. Mamysheva, "Pulse-overlapped dispersionmanaged data transmission and intrachannel four-wave mixing," Opt. Lett., vol. 24, pp. 1454-1456, 1999.

[3] R. I. Killey et al., "Reduction of intrachannel nonlinear distortion in 40-Gb/s-based WDM transmission over standard fiber," IEEE Photon. Technol. Lett., vol. 12, no. 12, pp. 1624-1626, Dec. 2000.

[4] E. A. Golovchenko et al., "Transmission properties of chirped return-tozero pulses and nonlinear intersymbol interference in $10 \mathrm{~Gb} / \mathrm{s}$ WDM transmission," in Proc. OFC/IOOC 2000, Baltimore, MD, Paper FC3.

[5] J. Mårtensson et al., "Timing jitter owing to intrachannel pulse interactions in dispersion-managed transmission systems," Opt. Lett., vol. 26, pp. 55-57, 2001.

[6] A. Mecozzi et al., "Cancellation of timing and amplitude jitter in symmetric links using highly dispersed pulses," IEEE Photon. Technol. Lett., vol. 13, no. 5, pp. 445-447, May 2001.

[7] O. V. Sinkin et al., "Calculation of error probability in WDM RZ systems in presence of bit-pattern-dependent nonlinearity and of noise," in Proc. OFC 2004, Los Angeles, CA, Paper TuN4.

[8] H. Xu et al., "Quantitative experimental study of intrachannel nonlinear timing jitter in a 10-Gb/s terrestrial WDM return-to-zero system," IEEE Photon. Technol. Lett., vol. 16, no. 1, pp. 314-316, Jan. 2004.

[9] R. J. Runser et al., "Recent results from ATDNet: An operating transparent network," in LEOS 2002, Glasgow, Scotland, Paper WF2.

[10] D. Marquis, "Bossnet: An all-optical long haul networking testbed," in LEOS 2000, Rio Grande, PR, Paper MC1.

[11] R. Holzlöhner et al., "Experimental and theoretical characterization of a $40 \mathrm{~Gb} / \mathrm{s}$ long-haul single-channel transmission system," J. Lightw. Technol., vol. 20, no. 7, pp. 1124-1131, Jul. 2002. 